\title{
Application of Nanoimprint Technology to Diffraction Grating Scale for Microrotary Encoder
}

\author{
Toshihiro Takeshita*, Takuma Iwasaki, Eiji Higurashi ${ }^{1}$ and Renshi Sawada \\ Graduate School of Systems Life Sciences, Kyushu University, \\ 744 Motooka, Nishi-ku, Fukuoka 819-0395, Japan \\ ${ }^{1}$ Research Center for Advanced Science and Technology, The University of Tokyo, \\ 4-6-1 Komaba, Meguro-ku, Tokyo 153-8904, Japan
}

(Received June 13, 2013; accepted September 2, 2013)

Key words: nanoimprint, optical MEMS, rotary encoder, diffracting grating

Using nanoimprint technology, we developed a rotary diffraction grating scale, which is used for microrotary encoding. The off-center error between the center of the throughhole for inserting a rotational axis and the center of the high-precision micropattern on the periphery of the scale is less than $3 \mu \mathrm{m}$ because we are able to shape the through-hole and the grating pattern simultaneously. The rotary grating is of sufficient accuracy to use as the scale in a microrotary encoder. The use of nanoimprinting is groundbreaking, in view of the traditionally poor centering precision of grating scale through-holes fabricated by conventional photolithography coupled with the machining of throughholes.

\section{Introduction}

Rotary encoders that use a diffraction grating(1) as the encoder scale are now a commercial reality and are embedded into devices such as motors and rotational stages. With the recent developments and increasing demand for highly functional miniaturized machines in robotic, medical, automotive, and space applications, a small rotary encoder is necessary. The size of a rotary encoder is decided by the encoder chip and grating scale. We studied a method of fabricating a rotary diffraction grating for use in a rotary encoder. In the conventional fabrication of a rotary diffraction grating scale, micropatterns of the grating scale are shaped by photolithography, after which a throughhole for the rotating axis is formed by cumbersome mechanical centering. Therefore, the off-center error between the center of the through-hole and the center of the micropatterns around the periphery of the grating scale can be significant. Additionally, the production process is not efficient because centering requires skill.

*Corresponding author: e-mail: takeshita@nano-micro.mech.kyushu-u.ac.jp 
In view of the above shortcomings, we developed a rotary diffraction grating scale for use in a microrotary encoder using nanoimprint technology ${ }^{(2-4)}$ applied to a microelectromechanical systems (MEMS) encoder chip..$^{(5-15)}$ The scales for a rotary encoder using imprint lithography have been reported. ${ }^{(16)}$ By applying nanoimprint technology, we can successfully form micropatterns by lithography and machine through-holes at the same time. Moreover, the off-center error is less than $3 \mu \mathrm{m}$.

Structures formed using nanoimprint technology are part of a promising trend of new MEMS structures to replace their silicon counterparts. Nanoimprint technologies include thermal nanoimprint lithography and UV nanoimprint lithography. We adopted thermal nanoimprint lithography for this task because it is better suited for low-cost mass production than its UV equivalent. However, generally, the transcription of precision patterns over a single polymer body with a high thermal expansion poses a serious problem that can impair the accuracy of precision patterns, owing to thermal expansion that follows the change in operating temperature. Polymers such as polycarbonate (PC) and polymethyl methacrylate (PMMA) are not suitable for high-precision positioning scales owing to their large coefficient of thermal expansion (CTE). However, in the case of rotational encoder scales, polymers can be used without problems since the thermal expansion of the scale is almost independent of the precision of the angular measurement during rotation. This is because the resolution of angular measurements is determined by the number of patterns on the full circle of the scale. Although the length of one pitch may be expanded owing to thermal expansion, the resolution of the rotating angular measurement does not change.

In this paper, we present the fabrication process for a rotational diffraction grating scale, its characterization, and its application in a MEMS encoder.

\section{Rotary Diffraction Grating Scale}

A scale formed by gratings on the periphery is used for the measurement of rotating angle through the measurement of the relative displacement against a rotary encoder. Figure 1 shows the structure of a reflective optical rotary encoder. The accuracy of the measurement of rotating angle can deteriorate if the center of rotation of the revolving scale, the center of the grating pattern (configured in a thin toroidal fashion), and the center of the through-hole provided for the insertion of the rotational axis deviate from each other, even slightly. In this regard, toroidal grating patterns and the through-hole center must be precisely aligned. In addition, the gap between a rotary encoder and the peripheral rotating scale must be maintained at a certain predetermined value. For this reason, it is preferable that a grating scale be flat and free of deformation, requiring at least an axisymmetric configuration.

The design of the diffraction grating scale is as follows: the scale is $12 \mathrm{~mm}$ in diameter and $0.6 \mathrm{~mm}$ in thickness. The diameter of the through-hole is $3 \mathrm{~mm}$, and it is formed at the center of the scale. Concavo-convex grating patterns $300 \mu \mathrm{m}$ in width, 0.4 $\mu \mathrm{m}$ in height, and $3.2 \mu \mathrm{m}$ in periodical pitch are formed on a grating scale at 6.4 and 10.6 $\mathrm{mm}$ in the radial direction. The numbers of patterns are 10125 and 6000, respectively. The smaller the error between the center of the gratings and the center of the rotation 


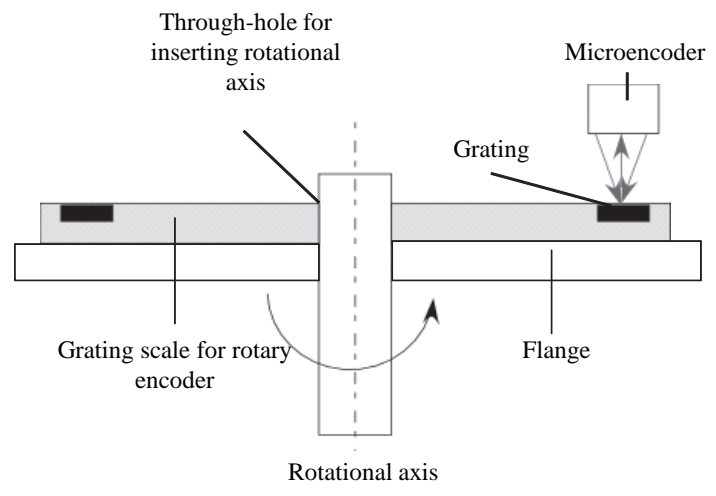

Fig. 1. Reflective optical rotary encoder.

or through-hole, the higher the precision of the measured rotating angles. Since we are developing MEMS encoder chips that are compact and highly precise, the decentering error should be small enough to fulfill these aims.

\section{Fabrication Process}

The diffraction grating manufacturing process is shown in Fig. 2. A stamper on which miniature concavo-convex patterns are formed at the correct height (taking thermal expansion into consideration) is attached to a piston. This miniature pattern is formed by photolithography. An electric heater is mounted on the piston and the outerdiameter (OD) blanking die. The air pressure on the back of the piston can be changed. The inner diameter (ID) blanking punch and OD blanking die are aligned concentrically. The hole is formed at the center of the stamper and has minimal clearance ( $3 \mu \mathrm{m})$ so that the ID at the blanking punch can slide the piston. The ID blanking punch, OD blanking die, and OD blanking punch-cum-ID blanking die are all precisely positioned using the guide posts and guide bushes located at the four corners. The blank substrate is formed from PC whose glass-transition temperature is about $150{ }^{\circ} \mathrm{C}$. The surface roughness of the blank substrate is $0.002 \mu \mathrm{m}$. The fabrication process flow is as follows.

1st step: The blank substrate is set on the stripper. Low-pressure air fills the back of the piston.

2nd step: The upper ram descends to touch the blank substrate and stamper. By keeping this position, the heaters attached in the OD blanking punch and the piston are activated, and the substrate is heated to $185^{\circ} \mathrm{C}$.

3rd step: After heating the substrate, the upper ram descends to the bottom dead point. This motion punches the substrate with the OD and ID blanking dies; the outline and through-holes are shaped simultaneously. Then, the punched substrate moves up and is tucked between the stamper and the OD blanking punch. After the upper ram reaches the bottom dead point, high-pressure air $(2.5 \mathrm{MPa})$ fills the back of the piston in order to 

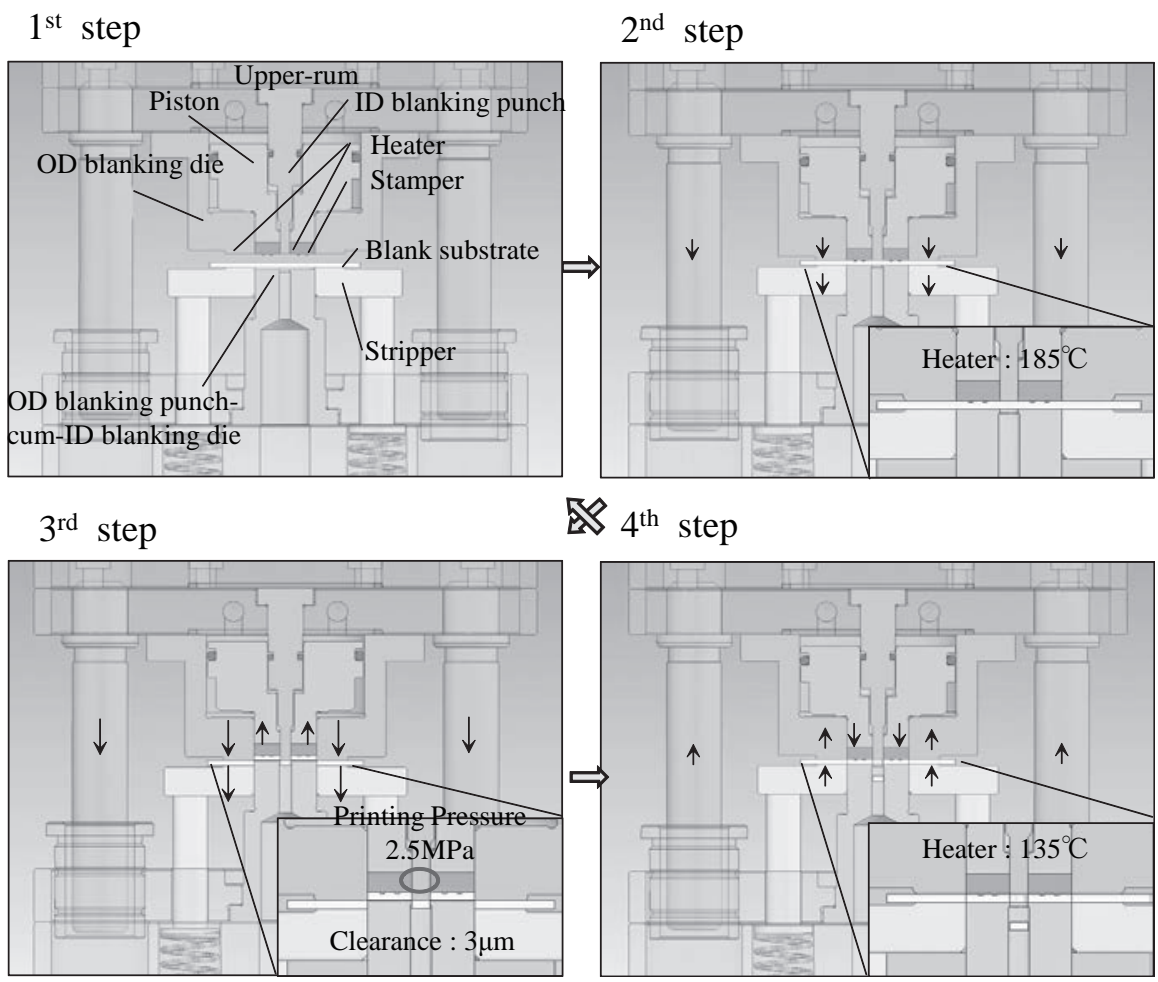

$4^{\text {th }}$ step

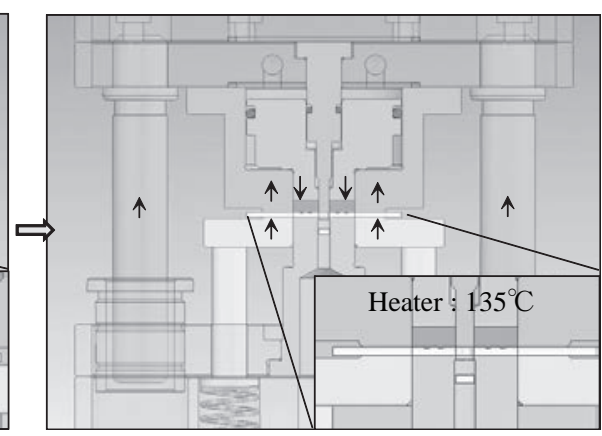

Fig. 2. Fabrication process.

imprint the miniature concave-convex patterns on the blank substrate. It takes $180 \mathrm{~s}$ to imprint the patterns, and the imprinting and shaping of the through-holes are carried out at the same time.

4th step: After the imprinting process, we cool the heaters of the OD blanking die and the stamper to $135{ }^{\circ} \mathrm{C}$ using cooling air, and lift up the upper ram. Lastly, we remove the punched blank substrate and the shaped substrate.

The maximum possible off-center alignment between the center of the gratings and the center of the through-hole is determined by the clearance. The decentering of the through-holes and the grating concentricity are within $3 \mu \mathrm{m}$ of the predetermined clearance.

The fabricated diffraction grating scale and a scanning electron microscopy (SS-550, Shimadzu corp.) image of the edge of the concave-convex pattern are shown in Figs. 3 and 4, respectively. To fabricate the release of the stamper and polymer easer, tapered shapes are formed at the edge of the convex pattern. 

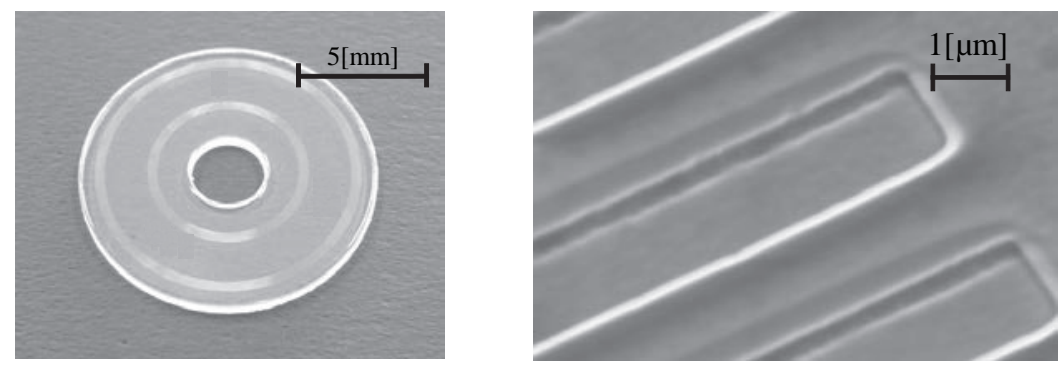

Fig. 3 (left). Picture of diffraction grating scale.

Fig. 4 (right). SEM image of concave-convex pattern.

\section{Characteristics}

\subsection{Geometry}

To confirm that the diffraction grating conforms to the intended design, we measured the geometry of the grating using a laser microscope. Figure 5 shows the results for one part of the grating. From this result, the height of the diffraction grating is $0.378 \mu \mathrm{m}$ and the periodical pitch is $3.226 \mu \mathrm{m}$. The grating is $0.4 \mu \mathrm{m}$ in height with a periodical pitch of 3.2 $\mu \mathrm{m}$, so mold patterns are printed on the polymer with high accuracy. The diffraction efficiency can be increased by increasing the aspect ratio. ${ }^{(17)}$ The accuracy can also be increased using an order of diffraction higher than the first order. ${ }^{(18)}$

The off-center error and warpage of the diffraction grating scale decrease the accuracy of the encoder. The off-center error causes radial runout when the grating rotates, and warpage causes the wobbling of the concavo-convex pattern in the vertical direction, which is known as face deflection. To measure the off-center error and warpage, a laser microscope (Wyko NT9300, Veeco) that can measure surface shape on the order of nanometers was used. The diffraction grating was placed on a flat plate and measured without inserting a rotational axis. The contour drawing of the grating is shown in Fig. 6(a). A large difference in height of $10 \mu \mathrm{m}$ or less is found across the whole surface of the grating. The cross-sectional shapes on the $x$-line, $y$-line, and $r$-line are evaluated as follows.

Figures 6(b) and 6(c) show cross-sectional drawings of the large differences in height on the $x$-line and $y$-line, respectively. In Fig. 6(a), xa1 and $x a 2$ indicate the $x$-coordinates of the central position of the outer diffraction grating pattern on the $x$-line, and $x b 1$ and $x b 2$ indicate the $x$-coordinates of the circumference of the through-hole on the $x$-line. $y a 1$ and $y a 2$ in Fig. 5(b) similarly indicate the $y$-coordinates of the center position of the outer diffraction pattern on the $y$-line, and $y b 1$ and $y b 2$ indicate the $y$-coordinates of the circumference of the through-hole on the $y$-line. The center of the grating pattern is thus (xac, $y a c)=(7.6591,6.5084)$ and the center of the through-hole is $(x b c, y b c)=(7.6607,6.5065)$. The off-center error is calculated using this equation.

$$
\text { Offcenter error }=\sqrt{(x a c-x b c)^{2}+(y a c-y b c)^{2}}=0.002484
$$




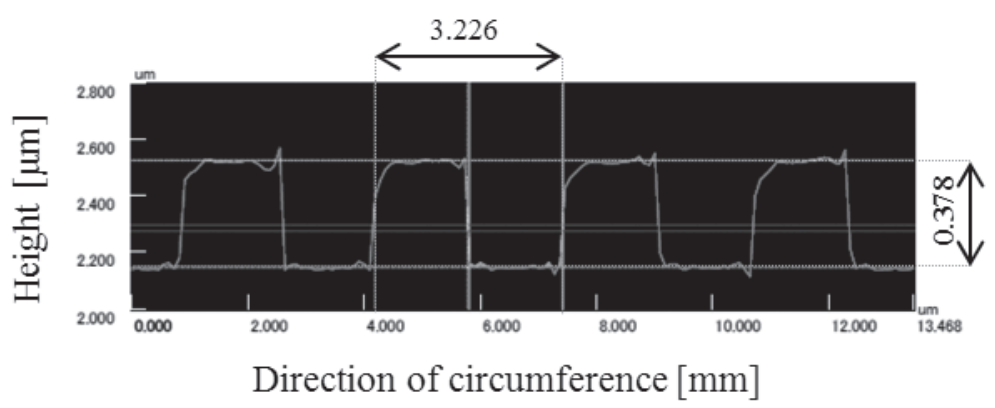

Fig. 5. Shape of concave-convex pattern.

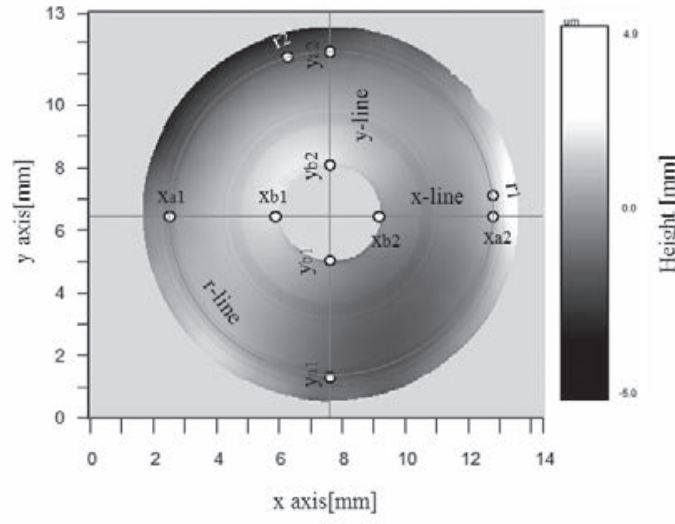

(a)

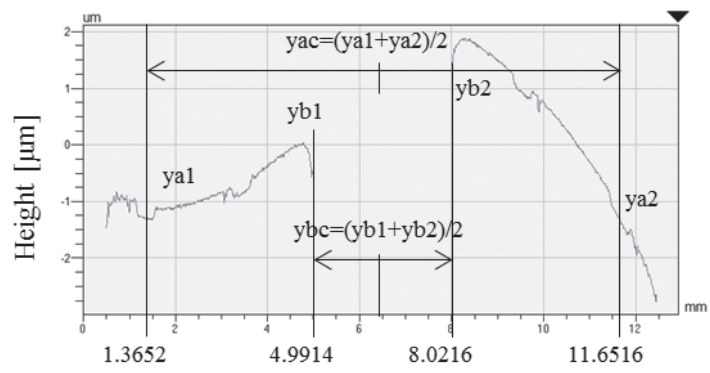

Distance $[\mathrm{mm}]$

(c)

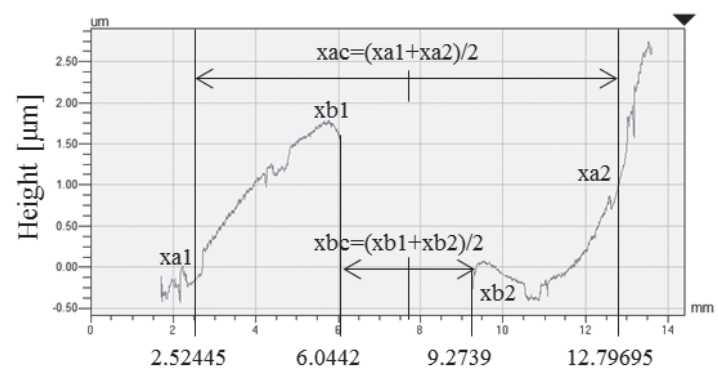

Distance $[\mathrm{mm}]$

(b)

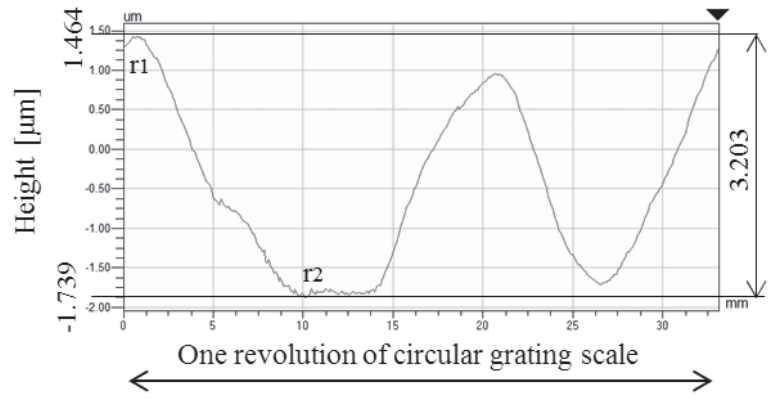

Distance $[\mathrm{mm}]$

(d)

Fig. 6. Contour drawing and cross-sectional drawings on three lines of diffraction grating scale: (a) contour drawing of diffraction grating scale, (b) cross-sectional drawings on $x$-line, (c) crosssectional drawings on $y$-line, and (d) cross-sectional drawings on $r$-line. 
The off-center error is only $2.484 \mu \mathrm{m}$ in our device, which is smaller than $3 \mu \mathrm{m}$, the clearance of the ID blanking punch and the piston.

Figure 6(d) shows cross-sectional drawings of the large difference in height on the $r$-line. The highest point is indicated by $r 1$, with a value of $1.464 \mu \mathrm{m}$. The lowest point is indicated by $r 2$, with a value of $-1.739 \mu \mathrm{m}$. As a result, the maximum difference in height is $3.203 \mu \mathrm{m}$. As the rotating axis is inserted into the through hole of this diffraction grating without inclination, the face deflection is about $3.20 \mu \mathrm{m}$ when the grating scale completes a 360 degree rotation. Although the difference in height is larger, by adhering to the use of the flange fabricated with a flatness of less than $3-5 \mu \mathrm{m}$, the face deflection is expected to decrease.

It is assumed that the warpage on the inner diffraction graining pattern is smaller than that on the outer diffraction grating pattern.

\subsection{Evaluation based on encoder signals}

We evaluated the characteristics of the fabricated diffraction grating scale by applying it to the MEMS encoder chip that we developed.

Figure 7 shows the diagram of the MEMS encoder chip that we developed. The sensor size is $2.8 \times 2.8 \mathrm{~mm}^{2}$ and $1.02 \mathrm{~mm}$ thick, and is fabricated using MEMS technology. The laser beams, which are emitted from both edge faces of a distributedfeedback (DFB) laser diode, are collimated using a glass lens after being reflected from a Au mirror in the cavity structure. The wavelength of the laser is $1.3 \mathrm{um}$. The phase of the laser beam is shifted by $90 \mathrm{deg}$. on passing through the film shifter. Both rays of the laser enter the concavo-convex pattern of the diffraction grating scale, and first-order diffraction beams interfere at the two photodiodes, i.e., PDA and PDB.

The experimental system is shown in Fig. 8. The diffraction grating scale is fixed on the flange of the rotating axis and is supported by a bearing. The MEMS encoder is fixed on jigs and aligned some distance from the diffraction grating, with the inclination controlled by a microstage. We obtain signals of IA and IB, the output signals from PD-A and PD-B, respectively, when the diffraction grating moves slightly relative to the MEMS encoder chip. Figure 9(a) shows IA and IB when the rotating axis rotates at a
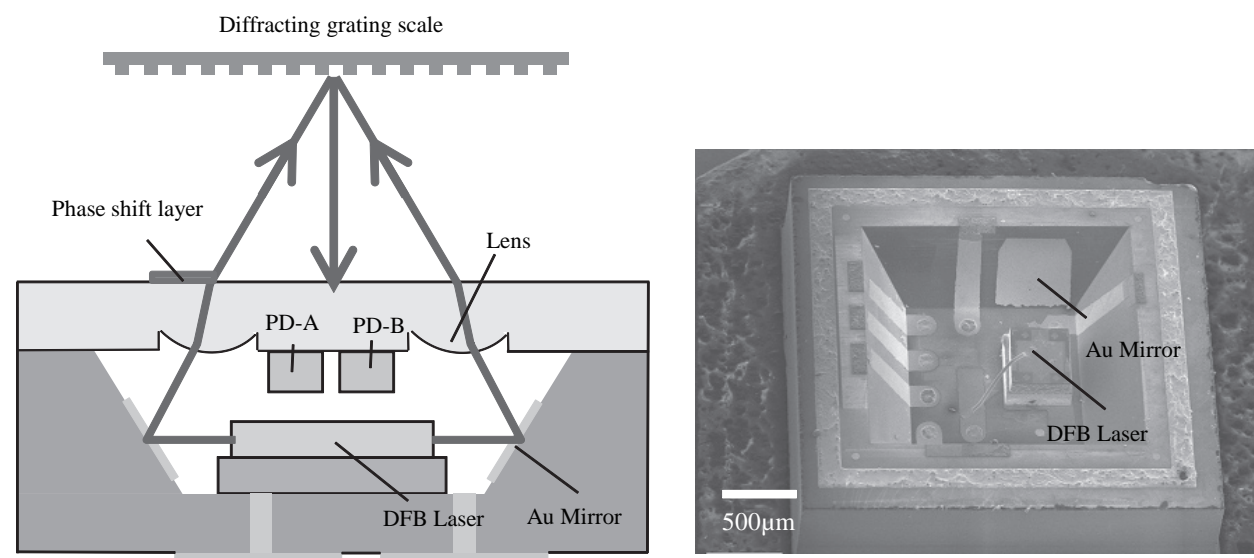

Fig. 7. MEMS microencoder chip. 


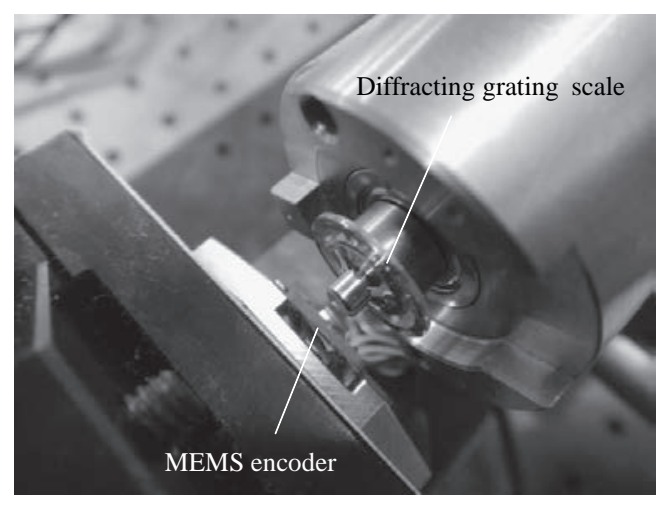

Fig. 8. Experimental system.

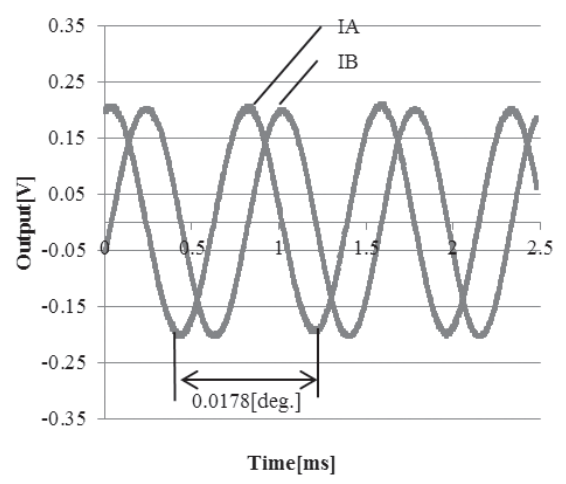

(a)

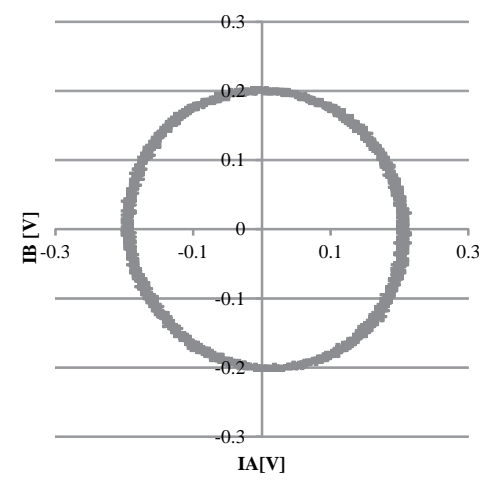

(b)

Fig. 9. Signals obtained using the fabricated polymer grating scale and the developed MEMS microencoder: (a) output signals of IA and IB and (b) Lissajous curve of IA and IB.

constant speed. Good sine curves are obtained. When the diffraction grating moves by one pitch, two pitch sine curves are obtained for IA and IB. There are 10125 concaveconvex patterns on the outer diffraction grating. Therefore, the number of pitches in the output signal is 20250, and one period of the signal corresponds to a rotation angle of 0.0178 deg. The Lissajous curve of IA and IB is shown in Fig. 9(b). The horizontal axis shows IA and the vertical axis shows IB. Because the two signals of IA and IB are phase-shifted by almost 90 deg., the Lissajous curve becomes circular.

From this result, it is demonstrated that nanoimprinting can be achieved with accuracy and that the diffraction grating scale can be used as the scale of a MEMS 
encoder chip. The resolution of the rotary encoder depends on the number of pitches in the grating pattern. When the grating scale completes one full revolution, 20250 pulses are generated as a digital signal. However, we process the output signal as analog. Therefore, the resolution is expected to increase further. Although the experimental peak-to-peak voltage is $0.4 \mathrm{~V}$, we expect to raise the voltage of the output by coating the diffraction grating with metal to raise the reflectivity.

\section{Conclusions}

We fabricated a diffraction grating scale for a rotary encoder using nanoimprint technology. Because the through-hole and micropattern are shaped simultaneously in our process, it is not necessary to perform cumbersome centering of the through-hole and micropatterns, which require skill. This is expected to enable the production of large volumes of the diffraction grating scale.

We measured the shape of the diffraction grating scale. The off-center error between the through-hole center and the center of the outer micropattern on the periphery of the scale is within $2.49 \mu \mathrm{m}$. Also, the difference in height on the outer micropattern, which may be caused by thermal expansion of the scale material, is less than $3.21 \mu \mathrm{m}$. Radial runout and face deflection of the rotary encoder diminish the accuracy of the rotation angle. The radial runout and face deflection in the MEMS encoder chip are less than 3 and $5 \mu \mathrm{m}$, respectively. These results prove that this rotary grating scale is sufficiently accurate for use in a microrotary encoder.

We have also demonstrated that the concave-convex pattern is shaped with high accuracy using nanoimprinting. We obtained good sine curve output signals from two PDs and a good Lissajous curve using the MEMS encoder.

\section{References}

1 G. N. Rassudova and F. M. Gerasimov: Opt. Spectrosc. 11 (1961) 136.

2 S. Y Chou, P. R. Krauss and P. J. Renstrom: Science 272 (1996) 85.

3 J. Mohr, B. Anderer and W. Ehrfeld: Sens. Actuators, A 27 (1991) 571.

4 S. Amaya, D. V. Dao, and S. Sugiyama: J. Micro/Nanolithogr. MEMS MOEMS 8 (2009) 4.

5 R. Sawada, E. Higurashi and Y. Jin: J. Lightwave Technol. 21 (2003) 815.

6 R. Sawada, E. Higurashi, T. Ito and I. Ishikawa: Sens. Actuators, A 136 (2007) 161.

7 E. Higurashi, T. Suga and R. Sawada: 9th Annual IEEE Conf. Sensors (IEEE Sensors 2010) (Waikaloa, Hawaii, November 1-4, 2010) p. 2409.

8 S. Nakamura, D. Chino, E. Higurashi, T. Suga and R. Sawada: Int. Conf. Electronics Packaging (ICEP 2009) (Kyoto, Japan, April 14-16, 2009) p. 971.

9 E. Higurashi, D. Chino, T. Suga and R. Sawada: Transducer 09 (Denver, June 21-25, 2009) W3p.031, p. 1873.

10 R. Sawada, E. Higurashi, A. Shimizu and O. Ohguchi: Technical Digest of IEEE/LEOS International Optical MEMS’2001 (Okinawa, Japan, September 25-28, 2001) p. 55.

11 R. Sawada, E. Higurashi, A. Shimizu, O. Ohguchi and M. Tsubamoto: Appl. Opt. 38 (2000) 6866.

12 R. Sawada, E. Higurashi, O. Ohguchi and Y. Jin: Proc. IEEE The Thirteenth MEMS’ 2000 (Miyazaki, Japan, 2000) p. 491. 
13 R. Sawada, H. Tanaka, O. Ohguchi, J. Shimada and F. Shimokawa: Int. J. Japan Soc. Prec. Eng. 28 (1994) 1.

14 R. Sawada: Digest of Technical Papers Transducers '95 (Stockholm, Sweden, 1995) p. 281.

15 R. Sawada, O. Ohguchi, K. Mise and M. Tsubamoto: Proc. IEEE MEMS’94 (January 25-28, 1994) 337.

16 M. Inokuchi, Y. Sakamoto, T. Abe, T. Akase, K. Dote and R. Sawada: Int. Microprocesses and Nanotechnology (MNC) (Fukuoka, October 27-30, 2008) 641.

17 K. Yokomori: Appl. Opt. 23 (1984) 2303.

18 E. Higurashi and R. Sawada: J. Micromech. Microeng. 15 (2005) 1459. 